\title{
Matérínia
}

ISSN 1517-7076

Revista Matéria, v. 11, n. 2, pp. 155 - 161, 2006

http://www.materia.coppe.ufrj.br/sarra/artigos/artigo10386

\section{Influencia de la Deficiencia de Itrio en la Sinterización de Cerámicos YBaCuO (123)}

\author{
E. Benavidez ${ }^{\mathrm{a}}$ y C.J.R. González Oliver ${ }^{\mathrm{b}, \mathrm{c}}$ \\ a. Facultad Regional San Nicolás, Universidad Tecnológica Nacional \\ Colón 332 - (2900) San Nicolás, Argentina. \\ e-mail: ebenavidez@frsn.utn.edu.ar \\ b. Centro Atómico Bariloche, C.N.E.A. \\ Av. Bustillo Km. 9,5 - (8400) Bariloche, Argentina. \\ c. CONICET \\ e-mail: gon@cab.cnea.gov.ar
}

\section{RESUMEN}

En este trabajo se analiza la densificación del cerámico superconductor $\mathrm{YBa}_{2} \mathrm{Cu}_{3} \mathrm{O}_{7-\mathrm{x}}$ (123) a partir de compactos conformados con dos composiciones deficientes en itrio con respecto a la relación catiónica superconductora $\mathrm{Y}: \mathrm{Ba}: \mathrm{Cu}=1,0: 2,0: 3,0$. La evolución térmica de los polvos se estudió por ATD-TG, en atmósfera de $\mathrm{O}_{2}$, hasta la zona de descomposición peritéctica del sistema $\mathrm{YBa}_{2} \mathrm{Cu}_{3} \mathrm{O}_{7-\mathrm{x}}\left(\approx 1050^{\circ} \mathrm{C}\right)$. La densificación de estos compactos se analizó por medio de dilatometrías realizadas bajo similares condiciones a las establecidas para los ATD-TG. Los mecanismos dominantes del sinterizado fueron analizados por medio de modelos aplicados a las curvas dilatométricas. De acuerdo a los datos combinados de dilatometrías y ATD-TG, se verifica que el mecanismo de sinterización por fase líquida (SFL) controla el $85 \%$ de la contracción total del compacto de composición $\mathrm{Y}_{0,7} \mathrm{Ba}_{2,0} \mathrm{Cu}_{3,3} \mathrm{O}_{\mathrm{x}}(\mathrm{C} 2)$, el cual presenta un temprano crecimiento de granos (CG) desde los $860^{\circ} \mathrm{C}$. El proceso de CG no se detecta en el compacto de composición $\mathrm{Y}_{0,7} \mathrm{Ba}_{2,3} \mathrm{Cu}_{3,1} \mathrm{O}_{\mathrm{x}}(\mathrm{C} 1)$ a temperaturas menores a los $900^{\circ} \mathrm{C}$. Para ambas composiciones se postula que, a partir de $900^{\circ} \mathrm{C}$, el mecanismo de SFL está presente por medio del proceso de reacomodamiento y luego, en el rango $930-975^{\circ} \mathrm{C}$, el mecanismo de solución-precipitación es el proceso dominante en el SFL.

Palabras claves: $\quad \mathrm{YBa}_{2} \mathrm{Cu}_{3} \mathrm{O}_{\mathrm{x}}$, sinterización, densificación, ATD-TG, dilatometría.

\section{Influence of Yttrium Deficiency on the Sintering of YBaCuO (123) Ceramics}

\section{ABSTRACT}

In this work the densification of $\mathrm{YBa}_{2} \mathrm{Cu}_{3} \mathrm{O}_{7-x}$ (or 123) ceramic starting from compacts with two compositions having lower $\mathrm{Y}$-contents relative to the superconducting cationic $\mathrm{Y}: \mathrm{Ba}: \mathrm{Cu}$ ratios equal to 1.0:2.0:3.0 were analyzed. Thermal evolution of the powders was monitored by DTA-TG, under $\mathrm{O}_{2}$-flow, up to the peritectic decomposition temperature, $\mathrm{Tp} \approx 1050^{\circ} \mathrm{C}$. The sintering/densification behavior of these compacts was followed by dilatometric runs under the same DTA-TG conditions. The main sintering mechanisms were analyzed using theoretical models fitted to the densification curves allowing the calculation of the apparent sintering enthalpies. According to dilatometric and DTA-TG runs, the liquid phase sintering (LPS) mechanism controls $85 \%$ of the total shrinkage in compact of composition $\mathrm{Y}_{0,7} \mathrm{Ba}_{2,0} \mathrm{Cu}_{3,3} \mathrm{O}_{\mathrm{x}}$ (C2) presenting an early grain growth (GG) contribution to densification from $860^{\circ} \mathrm{C}$. It should be noted no GG was detected in compact of composition $\mathrm{Y}_{0,7} \mathrm{Ba}_{2,3} \mathrm{Cu}_{3,1} \mathrm{O}_{\mathrm{x}}(\mathrm{C} 1)$ at temperatures lower than $900^{\circ} \mathrm{C}$. From $900^{\circ} \mathrm{C}$ the LPS is thought to be present by the rearrangement stage and then, in the range $930-975^{\circ} \mathrm{C}$, the solution-precipitation process in LPS would be the main densification mechanism.

Keywords: $\mathrm{YBa}_{2} \mathrm{Cu}_{3} \mathrm{O}_{\mathrm{x}}$, sintering, densification, DTA-TG, dilatometry. 


\section{INTRODUCCIÓN}

Es sabido que el grado de densificación de un cerámico influye de forma directa sobre las distintas propiedades del material. En el caso del cerámico superconductor $\mathrm{YBa}_{2} \mathrm{Cu}_{3} \mathrm{O}_{7-\mathrm{x}}$, también conocido como 123, es importante conocer su comportamiento de densificación durante el tratamiento térmico, ya que el porcentaje de porosidad que presenta este tipo de material tiene influencia directa sobre sus propiedades superconductoras [1] . Así, la densificación y el crecimiento de granos del cerámico $\mathrm{YBa}_{2} \mathrm{Cu}_{3} \mathrm{O}_{7-\mathrm{x}}$ define su microestructura, lo que a su vez, influye fuertemente en sus propiedades superconductoras.

En trabajos previos se analizó la densificación de compactos 123 con adiciones de Ag [2] y Sr [3]], donde se evalúo la aplicación de diferentes modelos de sinterizado sobre la curva de contracción.

Siguiendo con este tipo de estudio se busca analizar el comportamiento de densificación del sistema123 partiendo de compactos con diferentes composiciones químicas. En el presente trabajo se evalúan los mecanismos involucrados en la densificación de compactos cerámicos 123 con deficiencia de itrio respecto a la relación $\mathrm{Y}: \mathrm{Ba}: \mathrm{Cu}=1: 2: 3$. Comprobándose que la presencia de pequeñas desviaciones respecto a esta relación 1:2:3 relación provoca, previo a su descomposición peritéctica, importantes cambios en el comportamiento de su densificación. Esta deficiencia de itrio promueve la presencia, a partir de $925-935^{\circ} \mathrm{C}$, de un líquido que acelera notablemente la densificación del cerámico.

\section{EXPERIMENTAL}

Los polvos de partida fueron obtenidos a través del secado $\left(70^{\circ} \mathrm{C}, 24 \mathrm{~h}\right)$ de soluciones organometálicas formadas a partir de acetato de itrio, metoxietóxido de bario y butirato de cobre disueltos en solventes alcohólicos. Se diseñaron para este estudio dos composiciones deficientes en itrio respecto a la estequiométrica $\mathrm{Y}: \mathrm{Ba}: \mathrm{Cu}$ = 1:2:3 (denominadas $\mathrm{C} 1$ y C2). Ambos polvos presentaron un color verde intenso.

Los polvos ya secos fueron sometidos a un tratamiento de calcinación $\left(890^{\circ} \mathrm{C}, 12 \mathrm{~h}\right)$ bajo flujo de $\mathrm{O}_{2}$, y durante su etapa de enfriamiento se los sometió a un tratamiento intermedio de oxigenación $\left(460^{\circ} \mathrm{C}, 6 \mathrm{~h}\right)$. Ambos polvos transformaron a un color negro.

Análisis dispersivo en energía (EDS) fue empleado para determinar la composición de los polvos. Las relaciones Y:Ba:Cu determinadas para las composiciones fueron: $\mathrm{C} 1=0$, 7:2,3:3,1 y C2 = 0,7:2,0:3,3.

La identificación de las fases cristalinas presentes en cada uno de estos polvos de partida se realizó mediante difracción de rayos X (DRX) en un equipo Philips PW 1700.

La morfología de los polvos tratados fueron observados por microscopía electrónica de barrido (MEB) con un microscopio Philips 515.

Los ensayos de análisis térmico diferencial y termogravimetría (ATD-TG) fueron efectuados en forma simultánea en un equipo Netzsch STA-409. Las corridas fueron realizadas a una velocidad de calentamiento de $10^{\circ} \mathrm{C} / \mathrm{min}$ en atmósfera dinámica de $\mathrm{O}_{2}$.

Aproximadamente $50 \mathrm{mg}$ de cada tipo de polvo se prensó uniaxialmente (100 MPa) en una matriz cilíndrica. Las dimensiones típicas de las pastillas tuvieron 3,88 mm de diámetro y 1,09 mm de altura. Estos compactos, previo a los ensayos dilatométricos, fueron tratados a $750^{\circ} \mathrm{C}$, por 4 horas bajo flujo de $\mathrm{O}_{2}$, para eliminar componentes orgánicos. Luego de este tratamiento no se detectó ninguna modificación en las dimensiones de las muestras.

Las dilatometrías fueron realizadas sobre los compactos en condiciones similares a las establecidas para los ensayos de ATD-TG.

Los modelos de sinterización aplicados sobre las curvas dilatométricas obtenidas fueron detallados en trabajos previos $[\underline{2}, \underline{3}, \underline{8}]$. Básicamente, consistieron en evaluar las entalpías asociadas a distintos mecanismos responsables de la densificación de materiales cerámicos y vidrios, como ser: difusión en estado sólido (por volumen y por borde de grano), flujo viscoso y sinterización con asistencia de fase líquida.

\section{RESULTADOS}

Las figuras 1 y 2 muestran la morfología de los polvos C1 y C2, respectivamente, luego del tratamiento térmico a $890^{\circ} \mathrm{C}$ durante 12 horas en $\mathrm{O}_{2}$. Se observa que el polvo $\mathrm{C} 1$ presenta granos de tamaño menor a $1 \mu \mathrm{m}$, mientras que el polvo C2 exhibe granos del orden de los $2 \mu \mathrm{m}$, mostrando indicios de un incipiente crecimiento de granos.

De acuerdo a los DRX, en el polvo C1 se detectaron dos fases: 123 y 011, mientras que en el polvo $\mathrm{C} 2$ se detectaron tres fases:123, 011 y 001 (donde:123 $=\mathrm{YBa}_{2} \mathrm{Cu}_{3} \mathrm{O}_{\mathrm{x}}, 011=\mathrm{BaCuO}_{2}$ y $001=\mathrm{CuO}$ ). 


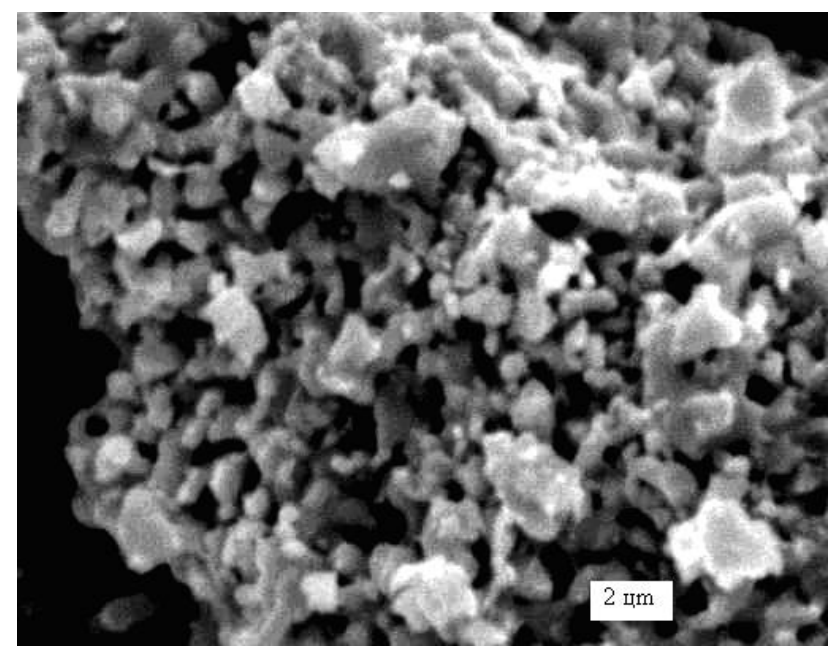

Figura 1: Micrografía de MEB del polvo C1. Barra $=2 \mu \mathrm{m}$.

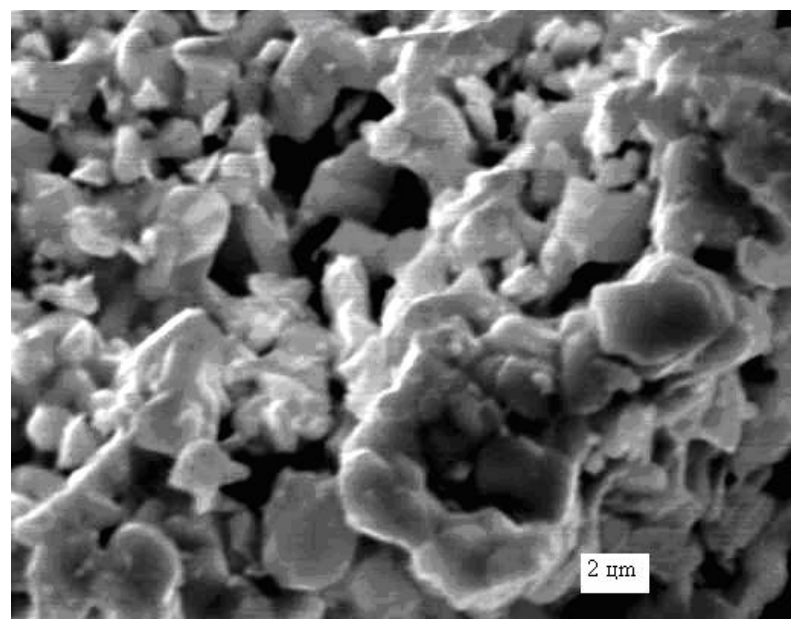

Figura 2: Micrografía de MEB del polvo C2. Barra $=2 \mu \mathrm{m}$.

Las curvas de ATD-TG de ambos polvos (Figuras 3 y 4) presentan varios picos endotérmicos previos a la Tp. Siguiendo trabajos previos [느므 se pueden considerar las siguientes reacciones $($ donde:211 = $\mathrm{Y}_{2} \mathrm{BaCuO}_{5}$ y $\left.202=\mathrm{Y}_{2} \mathrm{Cu}_{2} \mathrm{O}_{5}\right)$.

$$
\begin{aligned}
& \text { 925-935 }{ }^{\circ} \mathrm{C}: \quad \quad 123+011+001 \rightarrow \mathrm{L}(\mathrm{e} 1) \\
& \text { 963-975 }{ }^{\circ} \mathrm{C}: \quad 123+001 \rightarrow 211+\mathrm{L}(\mathrm{p} 1) \\
& \text { 995-1005 }{ }^{\circ} \mathrm{C}: \quad 211+001 \rightarrow 202+\mathrm{L}(\mathrm{p} 2) \\
& \text { 1012-1015 } \mathrm{C}: \quad 123+011 \rightarrow 211+(\mathrm{p} 3) \\
& 011 \rightarrow \mathrm{L}(\mathrm{m} 2) \\
& 211+011 \rightarrow \mathrm{L}(\mathrm{e} 3) \\
& \text { 1020-1037 }{ }^{\circ} \mathrm{C}: \quad 123 \rightarrow 211+\mathrm{L}(\mathrm{m} 1)
\end{aligned}
$$

En ambas figuras se muestran, además, las curvas dilatométricas de los compactos, con su respectiva derivada d $(\Delta \mathrm{L} / \mathrm{Lo}) / \mathrm{dT}$. En las mismas se observa que las mayores velocidades de contracción, para ambos compactos, se presentan en el rango $925-945^{\circ} \mathrm{C}$. 


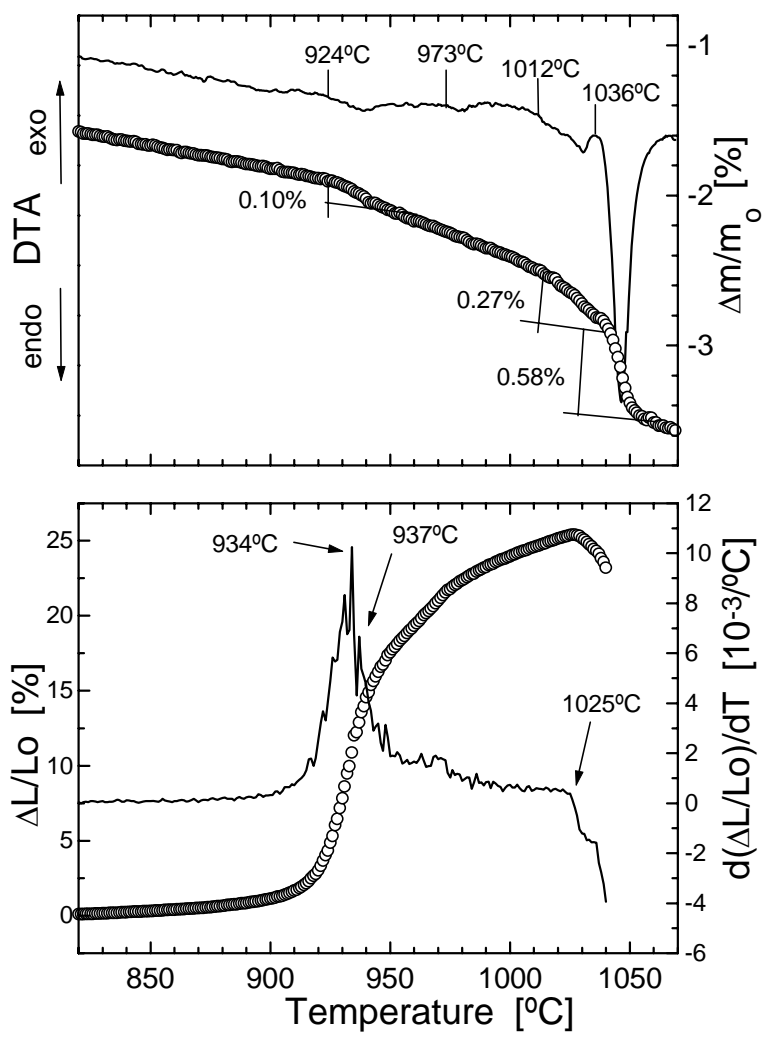

Figura 3: Curvas de ATD-TG y dilatométrica de 1a muestra C1.

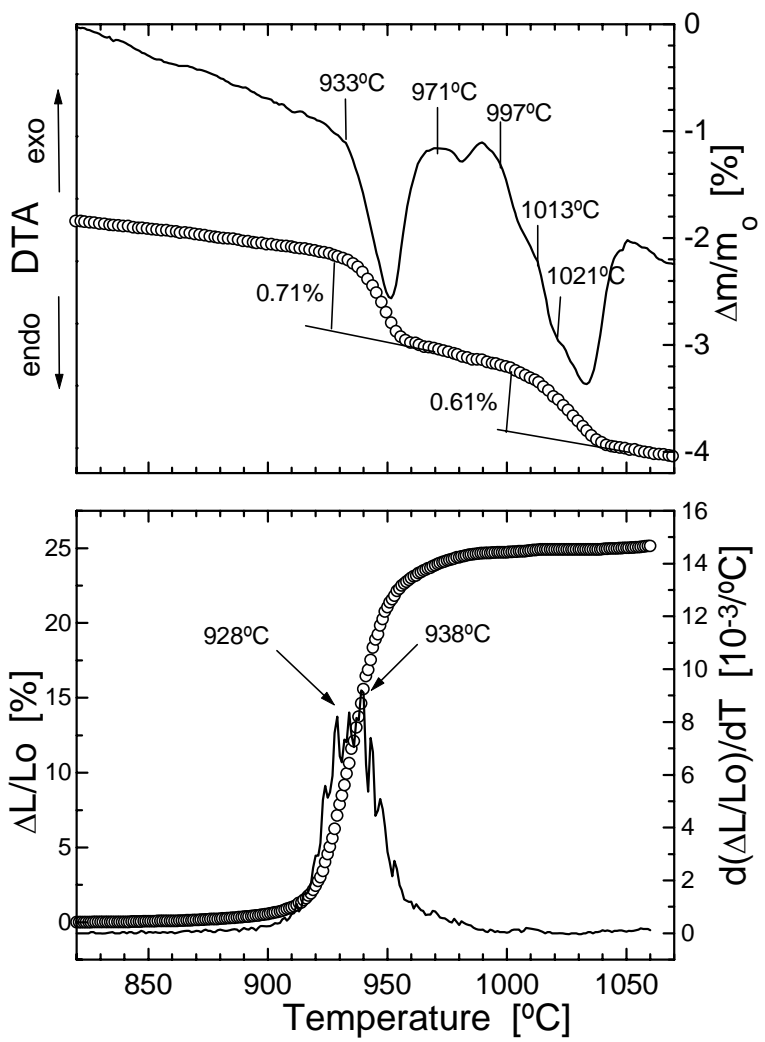

Figura 4: Curvas de ATD-TG y dilatométrica de la muestra C2. 
En las Tablas 1, 2 y 3, se presentan las entalpías de activación (Q) de los distintos modelos aplicados. En la Tabla 1, $Q_{b}{ }^{\text {ini }}$ y $Q_{v}{ }^{i n i}$, corresponden a las energías asociadas a los mecanismos de sinterización por difusión por volumen (subíndice $v$ ) y por borde de granos (subíndice $b$ ) correspondientes a la etapa inicial del sinterizado (supraíndice ini).

Tabla 1: Energías de activación calculadas para la etapa inicial del sinterizado.

\begin{tabular}{|c|c|c|c|c|}
\hline Muestra & $\begin{array}{c}\text { Rango de } \\
\left.\text { ajuste [ }{ }^{\circ} \mathrm{C}\right]\end{array}$ & $\begin{array}{c}\Delta \mathrm{L} / \mathrm{Lo} \\
{[\%]}\end{array}$ & $\begin{array}{c}Q_{b}{ }^{i n i} \\
{[\mathrm{~kJ} / \mathrm{mol}]}\end{array}$ & $\begin{array}{c}Q_{v}{ }^{i n i} \\
{[\mathrm{~kJ} / \mathrm{mol}]}\end{array}$ \\
\hline $\mathrm{C} 1$ & $818-901$ & $0,1-1,2$ & 862 & 553 \\
\hline $\mathrm{C} 2$ & $863-909$ & $0,1-0,9$ & 1592 & 1061 \\
\hline
\end{tabular}

En la Tabla 2, se calculan las energías de activación (Q) de los mismos mecanismos, pero desarrollados para la etapa intermedia del sinterizado (supraíndice int), en esta etapa se tiene en cuenta el crecimiento de los granos cerámicos (CG).

Tabla 2: Energías de activación calculadas para la etapa intermedia de sinterizado.

\begin{tabular}{|c|c|c|c|c|}
\hline Muestra & $\begin{array}{c}\text { Rango de } \\
\text { ajuste [ }\left[{ }^{\circ} \mathrm{C}\right]\end{array}$ & $\begin{array}{c}\Delta \mathrm{L} / \mathrm{Lo} \\
{[\%]}\end{array}$ & $\begin{array}{c}Q_{b}{ }^{i n t} \\
{[\mathrm{~kJ} / \mathrm{mol}]}\end{array}$ & $\begin{array}{c}Q_{v}{ }^{i n t} \\
{[\mathrm{~kJ} / \mathrm{mol}]}\end{array}$ \\
\hline $\mathrm{C} 1$ & $897-934$ & $1,0-10,9$ & 1208 & 1323 \\
\hline $\mathrm{C} 2$ & $863-901$ & $0,6-7,1$ & 430 & 434 \\
& $901-923$ & $0,6-3,4$ & 1423 & 1462 \\
\hline
\end{tabular}

La Tabla 3 presenta las energías calculadas aplicando modelos que consideran la densificación con presencia de fase líquida. Este mecanismo involucra dos etapas, primero la de reacomodamiento de partículas (rea) y luego la de solución-precipitación $(s-p)$.

Tabla 3: Energías de activación calculadas con el modelo de sinterización por fase líquida.

\begin{tabular}{|c|c|c|c|c|}
\hline Muestra & $\begin{array}{c}\text { Rango de } \\
\left.\text { ajuste [ }{ }^{\circ} \mathrm{C}\right]\end{array}$ & $\begin{array}{c}\Delta \mathrm{L} / \mathrm{Lo} \\
{[\%]}\end{array}$ & $\begin{array}{c}Q^{\text {rea }} \\
{[\mathrm{kJ} / \mathrm{mol}]}\end{array}$ & $\begin{array}{c}Q^{s-p} \\
{[\mathrm{~kJ} / \mathrm{mol}]}\end{array}$ \\
\hline \multirow{2}{*}{ C1 } & $897-934$ & $1,0-10,9$ & 1203 & - \\
& $930-941$ & $7,8-14,9$ & - & 715 \\
& $950-973$ & $17,5-21,5$ & - & 192 \\
\hline C2 & $901-930$ & $0,6-7,9$ & 1447 & - \\
& $932-048$ & $10,6-19,8$ & - & 814 \\
\hline
\end{tabular}

En todas las tablas se coloca el intervalo de temperatura donde fue realizado el ajuste (Rango de ajuste) y el porcentaje de contracción involucrado $(\Delta \mathrm{L} / \mathrm{Lo})$.

\section{ANÁLISIS Y DISCUSIÓN}

De acuerdo a la curva de dilatometría, el compacto de composición C1 inicia su contracción a partir de $790^{\circ} \mathrm{C}$ y desarrolla su etapa inicial de sinterizado en el rango $818-901^{\circ} \mathrm{C}$ con una $Q_{v}{ }^{i n i}$ de $553 \mathrm{~kJ} / \mathrm{mol}$. Por otro lado, el compacto C2 inicia su contracción a una temperatura superior $\left(827^{\circ} \mathrm{C}\right)$ y una $Q_{v}{ }^{i n i}$ también mayor $(1061 \mathrm{~kJ} / \mathrm{mol})$ que es dominante en el rango entre $863-909^{\circ} \mathrm{C}$. Sin embargo, debe destacarse que en dicho intervalo de temperaturas: $863-901^{\circ} \mathrm{C}$, el ajuste que considera el crecimiento de grano (etapa intermedia del sinterizado) presenta una $\mathrm{Q}$ menor $\left(Q_{v}{ }^{\text {int }}=Q_{b}{ }^{\text {int }}=432 \mathrm{~kJ} / \mathrm{mol}\right)$. Por lo tanto, este mecanismo se presenta como el más probable a ocurrir. Además, esta tendencia al CG a temperaturas menores a los $900^{\circ} \mathrm{C}$ por parte del compacto $\mathrm{C} 2$, se pone de manifiesto en las imágenes de MEB (Figuras 1 y 2), obtenidas sobre los polvo tratados a $890^{\circ} \mathrm{C}(12 \mathrm{~h})$. En dicha imagen el polvo C2 muestra indicios de operación del CG. 
Cabe mencionar, además, que capas de unos 2-5 $\mu \mathrm{m}$ de espesor con una composición química similar a la $\mathrm{C} 2$, fueron depositadas sobre substratos de titanato de estroncio $\left(\mathrm{SrTiO}_{3}\right)$ y tratadas 8 horas a $870^{\circ} \mathrm{C}$ en $\mathrm{O}_{2}$. Imágenes de MEB de la morfología desarrollada por dichas películas, confirman un crecimiento de los granos a estas temperaturas $[\underline{7}, \underline{8}]$.

Los modelos de densificación por reacomodamiento de granos originado por la presencia de líquido, ajusta muy bien en el compacto $\mathrm{C} 2$ cuando la temperatura supera los $900^{\circ} \mathrm{C}$. En este caso la energía de activación $Q^{\text {rea }}=1447 \mathrm{~kJ} / \mathrm{mol}$ en el rango $901-930^{\circ} \mathrm{C}$ y es del mismo orden que las calculadas para los mecanismos de difusión de estado sólido. Es muy probable que ambos mecanismos, de reacomodamiento y CG se estén desarrollando en forma simultánea en este rango. Luego, a partir de $932^{\circ} \mathrm{C}$ y hasta $948^{\circ} \mathrm{C}$ continúa una etapa de solución-precipitación $\left(Q^{s-p}=814 \mathrm{~kJ} / \mathrm{mol}\right)$ la cual se desarrolla hasta alcanzar una contracción del 20\%. Por lo tanto, alrededor del $85 \%$ de la contracción total de esta compacto se debe a la sinterización con ayuda de una fase líquida.

Lo expuesto está en acuerdo con un trabajo de Hwang et al. [9] donde se comprobó que una muestra rica en $\mathrm{CuO}$ promueve una mayor densificación debido a la presencia de una fase líquida. En nuestro caso, la presencia de fase líquida es atribuida a la reacción eutéctica (e1) que se presenta a 925-935 y genera el líquido L(e1), rico en Ba y Cu. Esta reacción tiene asociada una pérdida de masa $\Delta \mathrm{m} / \mathrm{m}_{\mathrm{o}}$ del $0,71 \%$ en el polvo C2.

Luego de los $950^{\circ} \mathrm{C}$ el compacto $\mathrm{C} 2$ ha alcanzado un valor elevado de la contracción $(\Delta \mathrm{L} / \mathrm{Lo} \approx$ $22 \%)$. A partir de $980^{\circ} \mathrm{C}$, la contracción se mantiene en un valor aproximadamente constante que oscila entre 24-25\%. De acuerdo a las curvas de ATD, se registran reacciones posteriores a esta temperatura previas a la Tp; sin embargo, no se observan posteriores modificaciones en la curva dilatométrica, por lo que se puede interpretar que los líquidos que se generan en la región entre $950^{\circ} \mathrm{C}$ y la Tp no producen ni contracción en el compacto ni exudado de líquidos.

El compacto C1 no desarrolla CG a temperaturas por debajo de los $900^{\circ} \mathrm{C}$. A mayor temperatura, los mecanismos de reacocomodamiento y CG por difusión de estado sólido, comparten el dominio del rango $900-930^{\circ} \mathrm{C}$, con una Q de unos $1200 \mathrm{~kJ} / \mathrm{mol}$. Entre $930-941^{\circ} \mathrm{C}$ la etapa de solución-precipitación es dominante con una $Q^{s-p}=715 \mathrm{~kJ} / \mathrm{mol}$, pero, en este caso, se interrumpe para volver a dominar en el rango 950-973 ${ }^{\circ} \mathrm{C}$ con una $Q^{s-p}$ considerablemente menor $(192 \mathrm{~kJ} / \mathrm{mol})$. El menor contenido de CuO en este polvo se cree que es responsable de la menor contracción $(\approx 15 \%)$ alcanzada por este compacto.

De la curva TG, se verifica una continua perdida de peso en el rango $950-1012^{\circ} \mathrm{C}$ con una $\Delta \mathrm{m} / \mathrm{m}_{0}$ del $0,20 \%$, provocado por las reacciones que generan los líquidos peritécticos $\mathrm{L}\left(\mathrm{p}_{2}\right)$ y $\mathrm{L}\left(\mathrm{p}_{3}\right)$. A temperaturas superiores a los $973^{\circ} \mathrm{C}$, ninguno de los modelos de densificación utilizados logra ajustar esta zona de temperaturas. Una probable explicación a esto, puede hallarse si se interpreta que los líquidos generados a estas temperaturas no promueven un fenómeno de solución-precipitación, por lo tanto no efectúan ningún aporte a la densificación del sistema.

Alrededor de los $1030^{\circ} \mathrm{C}$, el compacto C1 inicia un proceso de expansión. Este hecho puede ser explicado si se considera la reacción a $1010^{\circ} \mathrm{C}$ y la descomposición peritéctica, ambos generan una considerable cantidad de fase 211. La formación de esta fase en el sistema Y-Ba-Cu-O trae asociada una expansión considerable del volumen provocado por el coarsening de partículas $[\underline{10}, \underline{11}]$.

\section{CONCLUSIONES}

Los modelos aplicados en las diferentes etapas de la curva de densificación se correlacionaron muy bien con los datos de ATD-TG y las morfologías halladas en los polvos de ambas composiciones.

La deficiencia de itrio en el sistema YBaCuO-123 promueve la presencia, alrededor de los 925$935^{\circ} \mathrm{C}$, de un líquido eutéctico $\mathrm{L}(\mathrm{e} 1)$, rico en $\mathrm{Ba}$ y $\mathrm{Cu}$, que acelera notablemente la densificación del cerámico.

El polvo de composición $\mathrm{Y}_{0,7} \mathrm{Ba}_{2,0} \mathrm{Cu}_{3,3} \mathrm{O}_{x}$ (C2) mostró indicios de un crecimiento de granos a temperaturas tan bajas como $865^{\circ} \mathrm{C}$ en $\mathrm{O}_{2}$, lo cual es corroborado por los resultados de la aplicación de los modelos de densificación.

\section{AGRADECIMIENTOS}

Los autores desean agradecer a la Ing. S. Dutrús y al Sr. C. Cotaro por las imágenes de MEB y análisis de EDS y al Sr. D. Quattrini por los ensayos de ATD-TG, todos del Centro Atómico Bariloche. 


\section{BIBLIOGRAFIA}

[1] CHENG, C.W., ROSSE-INNES, A.C., ALFORD, Mc.N., HARMER, M.A., BIRCHALL, J.D., "The Effect of Porosity on the Superconducting Properties of $\mathrm{YBa}_{2} \mathrm{Cu}_{3} \mathrm{O}_{\mathrm{x}}$ Ceramics", Superconducting Science and Technology, v. 1, pp. 113-117, 1988.

[2] FISCINA, J.E., GONZÁLEZ OLIVER, C.J.R., ESPARZA, D., "The Sintering Kinetics of Ag$\mathrm{Y}_{1} \mathrm{Ba}_{2} \mathrm{Cu}_{3} \mathrm{O}_{7-\delta}$ Laminates and the Interfacial metal-ceramic Reaction”, Applied Superconductivity , v. 3, n. 5, pp. 277-287, 1995.

[3] GONZÁLEZ OLIVER, C.J.R., FISCINA, J.E., OLIBER, E.A., RUSSO, D., ESPARZA, D.A., "Kinetics of the Sintering of Superconducting $\mathrm{YBa}_{2-\mathrm{x}} \mathrm{Sr}_{\mathrm{x}} \mathrm{Cu}_{3} \mathrm{O}_{7-\mathrm{y}}$ ", Termochimica Acta, v. 203, pp. 353-360, 1992.

[4] ASELAGE, T., KEEFER, K., "Liquidus Relations in Y-Ba-Cu Oxides", Journal of Materials Research, v. 3, n. 6, pp. 1279-1291, 1988.

[5] LAY, K.W., RENLUND, G.M., "Oxygen Pressure Effect on the $\mathrm{Y}_{2} \mathrm{O}_{3}$-BaO-CuO Liquidus", Journal of the American Ceramic Society, v. 73, n. 5, pp.1208-1213, 1990.

[6] PleWA, J., DI BeNEDETTO, A., ALTENBURG, H., EBER, G., KUGELER, O., SCHMITZ, G.J., "Thermal Investigations of Reactive Powder Mixtures as Precursors for Melt-processing of $\mathrm{YBa}_{2} \mathrm{Cu}_{3} \mathrm{O}_{7-\mathrm{x}}$ ", Journal of Thermal Analysis, v. 48, pp. 1011-1026, 1997.

[7] BENAVIDEZ, E., GONZÁLEZ OLIVER, C.J.R., CARUSO, R., DE SANCTIS, O., "Chemical Method to Prepare $\mathrm{YBa}_{2} \mathrm{Cu}_{3} \mathrm{O}_{7-\mathrm{x}}$ (YBCO) Films by Dipping Onto $\mathrm{SrTi}(\mathrm{Nb}) \mathrm{O}_{3}$ Ceramics”, Materials Chemistry and Physics, v. 62, pp. 9-17, 2000.

[8] BENAVIDEZ, E.R., "Comportamiento a Altas Temperaturas de Películas Delgadas, Capas Gruesas, Polvos y Compactos en el Sistema Superconductor $\mathrm{YBa}_{2} \mathrm{Cu}_{3} \mathrm{O}_{7-\mathrm{x}}$, con y sin Dopaje de Plata”, D.Sc. Tese, Universidad Nacional de Rosario, Rosario, Argentina, 2001.

[9] HWANG, N.M., PARK, Y.K., LEE, H.K., HAHN, J.H., BAHNG, G.W., LEE, K.W., MOON, H.G., PARK, J.C., "Effects of a Liquid Phase in the Y-Ba-Cu-O Superconductor", Journal of the American Ceramic Society, v. 71, pp. C210-C211, 1988.

[10] GRIFFITH, M.L., HUFFMAN, R.T., HALLORAN, J.W., "Formation and Coarsening Behavior of $\mathrm{Y}_{2} \mathrm{BaCuO}_{5}$ from Peritectic Decomposition of $\mathrm{YBa}_{2} \mathrm{Cu}_{3} \mathrm{O}_{7-\mathrm{x}}$ ”, Journal of Materials Research, v. 9, n. 7, pp. 1633-1643, 1994.

[11] OLIBER, E.A., BENAVIDEZ, E.R., REQUENA, G., FISCINA, J.E., GONZÁLEZ OLIVER, C.J.R., "High Temperature Reactions, Densification and the Peritectic Decomposition of $\mathrm{YBa}_{2-\mathrm{x}} \mathrm{Sr}_{\mathrm{x}} \mathrm{Cu}_{3} \mathrm{O}_{7-\delta}$ (YBSCO) Superconducting Ceramics”, Physica C, v. 384, pp. 247-257, 2003. 